\title{
RFID Base Path Tracking of Industrial Mobile Robots Localization for Indoor Environment
}

\author{
Jesika $\mathbf{R}^{1}$, Saravanan $\mathbf{K}^{2}$ \\ PG scholar, Department of ECE, CMS College of Engineering and Technology, Coimbatore, India ${ }^{1}$ \\ Assistant Professor, Department of ECE, CMS College of Engineering and Technology, Coimbatore, India ${ }^{2}$
}

\begin{abstract}
Recently, academia and industry have focused on the creation of new systems for mapping and exploration of unknown and known spaces. Such networks comprise of sensor nodes and relay nodes carried by robots for information sensing and communication, and are able to perform accurate and real time inspection, especially in adverse environments. A practical dynamic Path Tracking (PT) scheme for industrial robots is presented. The robot localizes itself by means of a Kalman based algorithm that fuses robot odometry with the information coming from the phase of the signals of few reference RFID tags deployed along the path. Sensor node localization is critical in such networks because localization results can be used not only for locating and pinpointing leaks, but also for manoeuvring mobile sensor node's in a channel of complex configuration. The developed PT scheme can be seamlessly integrated with the industrial robot controller and improve the robot's accuracy without retrofitting with high-end encoder.
\end{abstract}

Keywords: Radio Frequency Identification (RFID), path tracking, sensor node and relay node.

\section{INTRODUCTION}

In industrial manufacturing fields, many tasks, such as cutting, milling, and lathing, are expected to use robots to implement operation automatically. According to the standard process specifications in aerospace industry (e. g, Airbus [1]), the desired accuracy of robot manipulation for manufacturing is around $\pm 0.20 \mathrm{~mm}$. However, due to the mechanical tolerances and deflection in the robot structure, the typical difference of a virtual robot in simulation and a real robot can be $8-15 \mathrm{~mm}$, which is inadequate to meet the precision requirements of many potential applications. Therefore, the relatively low accuracy of current robots is the main problem for the industrial manufacturing applications. Especially, it poses a critical obstacle to use advanced task planning techniques which integrate off-line simulation and CAD-based methods.

Once a building, a wall, or other objects block the satellites from the user's sight, the global navigation satellite system GNSS localization will be affected negatively or even fail. Despite this issue, the demand of path navigation and positioning in indoor environments is increasing in recent years, either for personal and equipment safety or for life conveniencein an underground coal mine, tacking miners quickly and accurately is of vital importance, especially in emergency situations. Similar examples can also be found in smart 'buildings, factories, libraries, and airports.

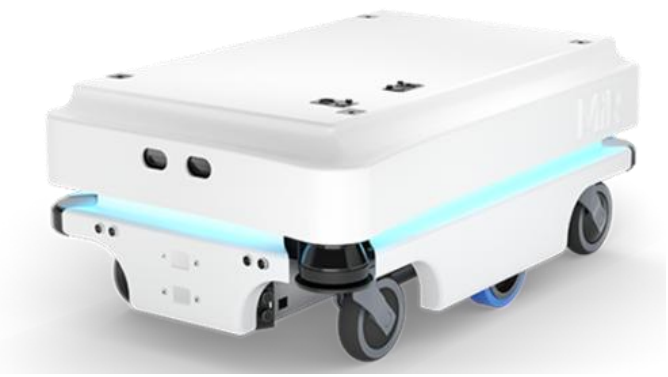

Fig.1. Industrial mobile robot.

Active LF RFID based systems are applied in several industrial and services sectors. They provide many advantages in particular in warehouse environments, where the automatic identification and positioning of goods make easy both storage and delivery. In this context, the localization of tagged objects and goods is an important application with a great economical value, while the trend for the future is the employment of LF RFID as enabling technology for the 
automatic manipulation of objects. A robot can recognize a tagged object by its ID, localize its position and pick it up in order to handle the object for a specific operation (e.g. packaging, assembling, and serving). The knowledge of the exact position of an RFID-tagged object is an open issue, several localization algorithms and procedures have been carried out in the literature but without a definitive solution. From the point of view of the application, the methods and the algorithms three classes: (i) localization and tracking of moving tagged goods, e.g. on a conveyor belt; (ii) localization or relative positioning of tagged goods standing on paths, e.g. a smart library; (iii) navigation of autonomous vehicles in smart environments, e.g. a robot that autonomously localizes both itself and the objects that it has to handle.

\section{PROPOSED SYSTEM}

The emerging new localization algorithms and methods based on Wireless Sensor Networks (WSN), permit to achieve a good trade-off between cost and localization accuracy especially in situations where WSN are already deployed for other purposes. Moreover, the hybridization of RFID and WSN technologies is possible and permits to increase the robustness and accuracy of positioning in many different environments. In this project, we consider the problem of a robot that patrols a warehouse and localizes tagged objects set on the paths by means of an RFID reader placed aboard and few reference tags deployed along the paths. Apart from these reference tags, the warehouse environment is unknown to the robot that determines its path exploiting only data collected from the odometry sensor and from the RFID readings: no other positioning sensor is used.

In fact, all data necessary for the localization are gathered using only two specific paths of the robot with respect to the paths while the 2D localization of objects on paths makes use of algorithms that exploit the properties of the phase pattern collected along the two paths, reducing the complexity of the searching. The two paths followed by the robot have been suitably conceived to decouple the problem of estimating the different coordinates of the tags, making

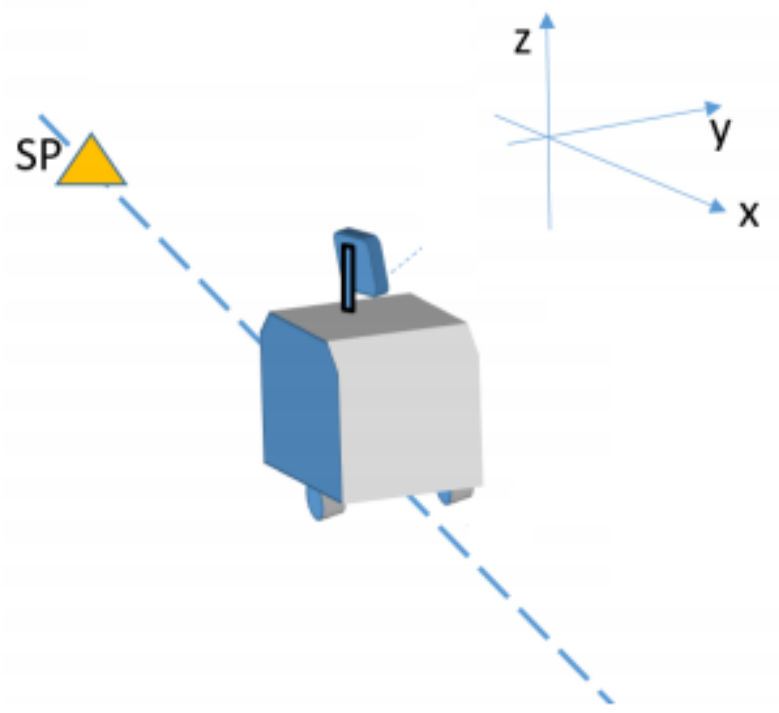

Fig.2. ISP: Robot Starting Position.

the estimation possible even under the unknown offset characterizing the RFID signal phases. The effectiveness of the proposed system has been checked experimentally obtaining results that outperform the cited literature in terms of localization accuracy. Furthermore the simplicity of the set-up and algorithms make it reliable and economically appealing.

The localization of tagged objects on the paths by means of an automatic vehicle like a robot requires the definition of a unique coordinate reference system in the real scenario. The frontal surface of the framework of the shelf and the direction orthogonal to that plane define a natural reference system as shown in Fig., where the xz plane coincides with the frontal surface of the shelf.

A couple of reference tags (T1, 2 and T3 in Fig.), placed at a known and arbitrary position on the framework of the shelf, permits to define a metric but more reference tags can be used to improve the localization accuracy and/or in case of large paths. The robot is equipped with an LF-RFID reader having an antenna appropriately oriented. During its motion, the robot collects odometry data and the phase of the signals backscattered by all the tags located on the shelf. The localization of the tagged objects is then accomplished through a two-step procedure. 


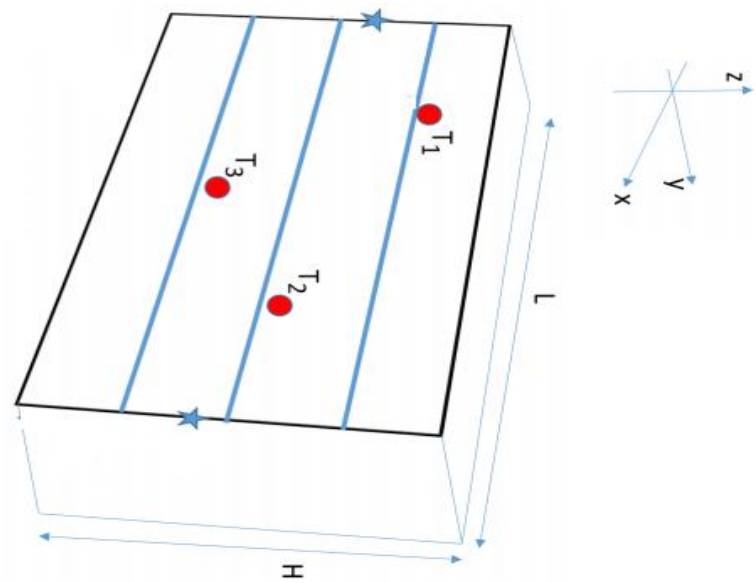

Fig.3. Referene tags objects.

In complex scenarios with multiple rows of long paths, the procedure described in this paper repeats equal to itself for each shelf (or portion of it in the case of long paths). In fact, because of the short range communication technology of UHF RFID, the reading distance is limited to few meters, compatible with the size of a single shelf (or portion of it). In this case the reference tags serve not only for the localization of the robot but also for the identification of the shelf. Additional reference tags could be placed in other parts of the environment to allow the robot motion among the rows of paths.

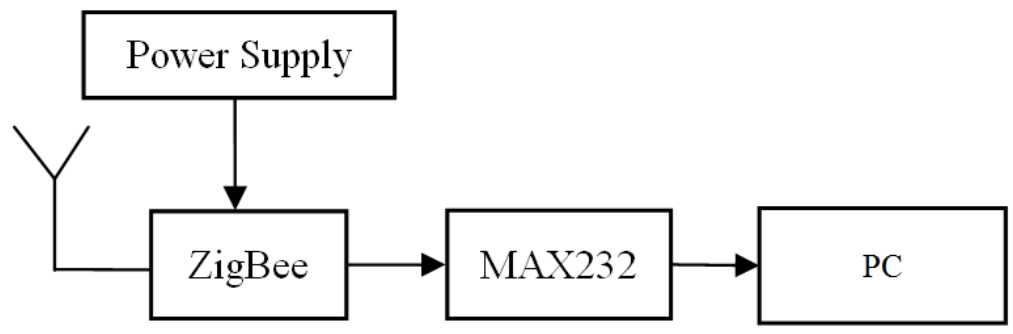

Fig.4. Block diagram of receiver.

A. $\quad$ Proposed description

- More recently, several applications based on ultrasonic technology have been developed to improve the mapping and localization.

- A robot module for the exploration of unknown and/or dangerous spaces that cannot be accessed by humans.

- As a matter of fact, ultrasonic sensors have become a popular measurement tool because of both their simplicity and affordability.

The direction is estimated by the angle between the two RFID tags which a robot passed one after another. The robot's accurate position information is needed when sensing a RFID tag to estimate the robot's direction precisely. But, with the RFID tags based localization method, a robot cannot find its accurate position (x,y). A robot just can determine whether it is near the position $\left(\mathrm{x}^{\prime}, \mathrm{y}^{\prime}\right)$ of the informed RFID tag in a certain distance. The distance error between the robot's real position and the estimated position is proportion to the standard deviation of the distance between the RFID tag and the RFID reader. So, we apply the localization to find the robot's position more accurately and Kalman filter to estimate the robot's direction. The accuracy of the position and the direction is related each other. We can raise the accuracy of the direction by raising the accuracy of the position, and estimate the robot's position in the area where the robot cannot sense the any RFID tags by estimating the robot's direction cannot sense the any RFID tags by estimating the robot's direction precisely. The advantage of RFID technology over bar codes or magnetic strips is that RFID doesn't need any physical contact as in case of magnetic strips (ATM or credit cards) or needn't be placed in a particular position as in case of bar codes. RFID is a wireless, non-contact type technology. Hence, it contains two components: RFID Cards or Tags and RFID Reader. RFID Reader Modules are basically radio frequency transceiver (transmitter and receiver). A simple RFID reader consists of an antenna, demodulator, data decoder unit and some filters. RFID readers can read and/or write data in to the RFID cards (depending on the type of the card). The RFID Reader module used in this project is EM - 18. It radiates a $125 \mathrm{KHz}$ signal through its antenna and hence a similar frequency based RFID Card must be used. The main components of the project are MCU, RFID Reader, some RFID 
cards and an alphanumeric LCD display. The design of the circuit is described here. As Arduino is the main processing device, all the connections are the connections are explained with respect to it. The RFID Reader module used in the project i.e. EM - 18 consists of 4 pins: Vcc, TX, RX and GND. As this module runs on 12V, it must be powered separately using a $12 \mathrm{~V}$ supply.

In this project, a MCU board is integrated with an RFID Reader Module in order to process the data or information retrieved from the RFID Card or Tag. The communication between the RFID Reader and external devices like microcontrollers is implemented using UART protocol (hence, TX and RX pins). As the reader reads the information from the card and transmits to the host device i.e. MCU, the TX pin of the Reader must be connected to RXD pin (Pin 0 ) of Arduino. The RFID reader module continuously emits electromagnetic radiation in the form of radio waves at a frequency of $125 \mathrm{KHz}$. When a passive RFID card is brought near this field, due to the concept of mutual induction, the electromagnetic field from the reader induces a small current in the antenna coil of the card. The xBee - series 1 modules take the 802.15.4 stack (the basis for Zigbee) and wrap it into a simple to use serial command set. These modules allow a very reliable and simple communication between micro controllers, computers or other systems by using just a serial port. They can communicate up to $300 \mathrm{Ft}(\sim 100 \mathrm{~m})$, they have $2.4 \mathrm{GHz}$ frequency, use the 802.15 .4 protocol and have data rate up to $250 \mathrm{kbps}$. They have also a $1 \mathrm{~mW}$ wire antenna on them. They supports Point to point and multi-point networks.

Input sensor emits an ultrasound at $40000 \mathrm{~Hz}$ which travels through the air and if there is an object or obstacle on its path It will bounce back to the module. Considering the travel time and the speed of the sound you can calculate the distance. The HC-SR04 Ultrasonic Module has 4 pins, Ground, VCC, Trig and Echo. The Ground and the VCC pins of the module needs to be connected to the Ground and the 5 volts pins on the Arduino Board respectively and the trig and echo pins to any Digital I/O pin on the Arduino Board. In order to generate the ultrasound you need to set the Trig on a High State for $10 \mu \mathrm{s}$. That will send out an 8 cycle sonic burst which will travel at the speed sound and it will be received in the Echo pin. The Echo pin will output the time in microseconds the sound wave travelled first you have to define the Trig and Echo pins. In this case they are the pins number 9 and 10 on the Arduino Board and they are named trigPin and echoPin. Then you need a Long variable, named "duration" for the travel time .

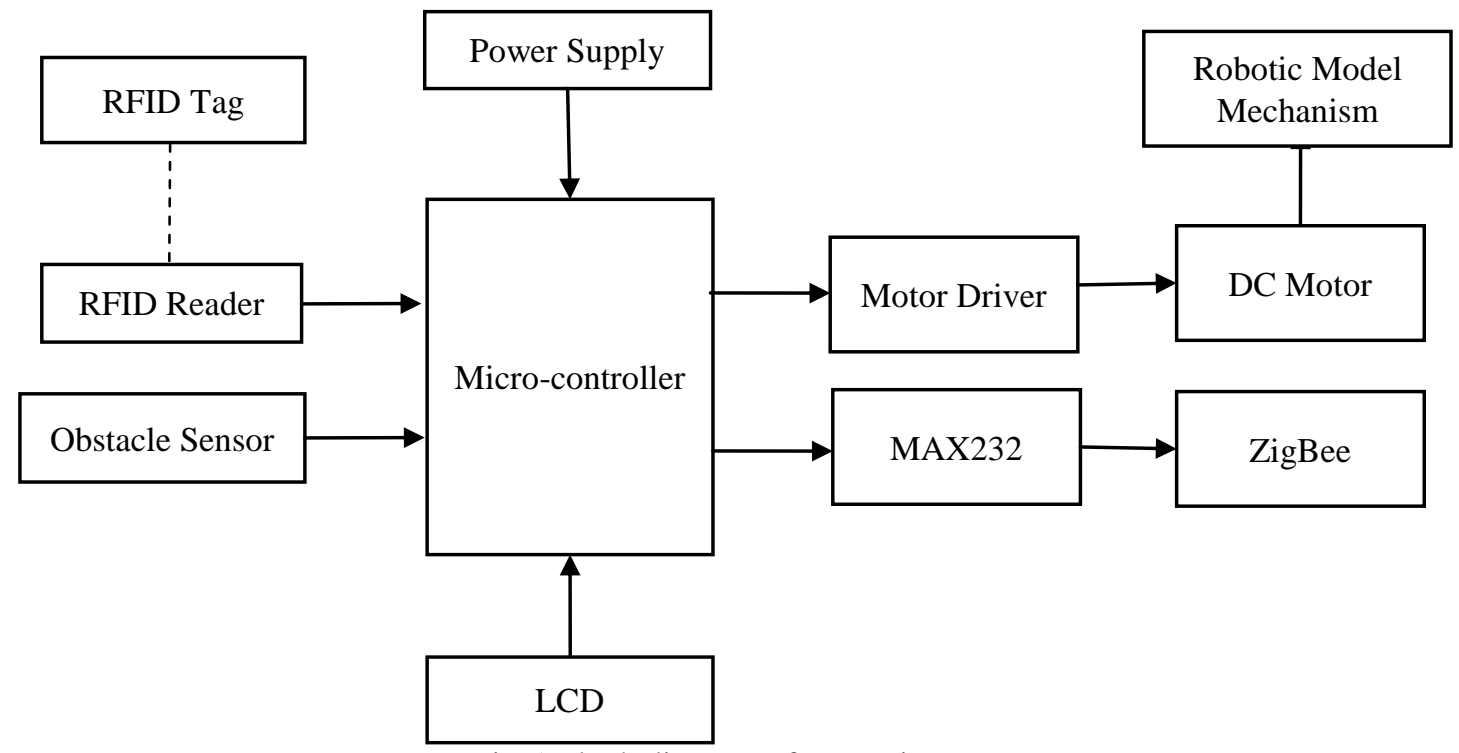

Fig 5.Block diagram of transmitter.

In the setup you have to define the trigPin as an output and the echoPin as an Input and also start the serial communication for showing the results on the serial monitor. The L293D is a 16-pin Motor Driver IC which can control a set of two DC motors simultaneously in any direction. The L293D is designed to provide bidirectional drive currents of up to $600 \mathrm{~mA}$ (per channel) at voltages from $4.5 \mathrm{~V}$ to $36 \mathrm{~V}$ (at pin 8!). You can use it to control small de motors - toy motors. Sometimes it can be extremely hot. The L293D IC receives signals from the microprocessor and transmits the relative signal to the motors. It has two voltage pins, one of which is used to draw current for the working of the L293D and the other is used to apply voltage to the motors. The L293D switches it output signal according to the input received from the microprocessor. The L293D is a 16 pin IC, with eight pins, on each side, dedicated to the controlling of a motor. There are 2 INPUT pins, 2 OUTPUT pins and 1 ENABLE pin for each motor. L293D consist of two Hbridge. H-bridge is the simplest circuit for controlling a low current rated motor. 
Vol. 8, Issue 4, April 2019

II. RESULT

ZigBee shows the Fig 6., is an IEEE 802.15.4-based specification for a suite of high-level communication protocols used to create personal area networks with small, low-power digital radios, such as for home automation, medical device data collection, and other low-power low-bandwidth needs, designed for small scale projects which need wireless connection. The technology defined by the ZigBee specification is intended to be simpler and less expensive than other Wireless Personal Area Networks (WPANs), such as Bluetooth or Wi-Fi. Applications include wireless light switches, electrical meters with in-home-displays traffic management systems, and other consumer and industrial equipment that requires short-range low-rate wireless data transfer. Its low power consumption limits transmission distances to 10-100 meters line-of-sight, depending on power output and environmental characteristics. ZigBee devices can transmit data over long distances by passing data through a mesh network of intermediate devices to reach more distant ones. ZigBee is typically used in low data rate applications that require long battery life and secure networking (ZigBee networks are secured by 128 bit symmetric encryption keys.) ZigBee has a defined rate of $250 \mathrm{kbit} / \mathrm{s}$, best suited for intermittent data transmissions from a sensor or input device. The MAX232 shows Fig 7., is an integrated circuit first created in 1987 by Maxim Integrated Products that converts signals from a TIA-232 (RS-232) serial port to signals suitable for use in TTL-compatible digital logic circuits. The MAX232 is a dual transmitter / dual receiver that typically is used to convert the RX, TX, CTS, RTS signals. The drivers provide TIA-232 voltage level outputs (about \pm 7.5 volts) from a single 5-volt supply by on-chip charge pumps and external capacitors. This makes it useful for implementing TIA-232 in devices that otherwise do not need any other voltages. The MAX232 replaced an older pair of chips MC1488 and MC1489 that performed similar RS-232 translation. The MC1488 quad transmitter chip required 12 volt and -12 volt power, and MC1489 quad receiver chip required 5 volt power. The main disadvantages of this older solution was the $+/-12$ volt power requirement, only supported 5 volt digital logic, and two chips instead of one.

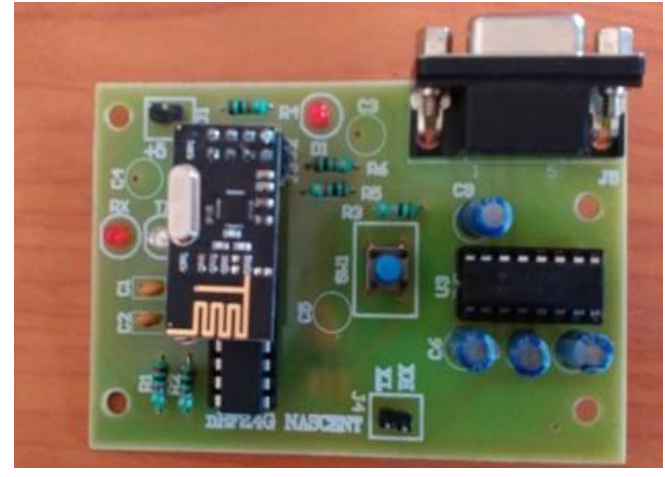

Fig.6. Zigbee.

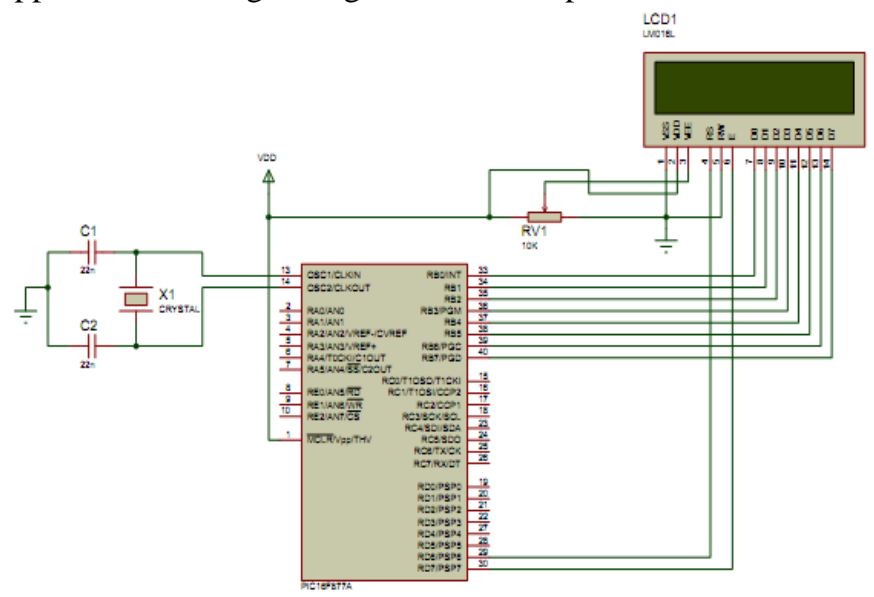

Fig.7. LCD interface with PIC16F877A.

LCD (Liquid Crystal Display) shows the Fig 8., screen is an electronic display module and find a wide range of applications. A 16x2 LCD display is very basic module and is very commonly used in various devices and circuits. A 16x2 LCD means it can display 16 characters per line and there are 2 such lines. In this LCD each character is displayed in 5x7 pixel matrix. This LCD has two registers, namely, Command and Data. The command register stores the command instructions given to the LCD, The data register stores the data to be displayed on the LCD. The data is the ASCII value of the character to be displayed on the LCD.Liquid crystal displays are used for display of numeric and alphanumeric character in dot matrix and segmental displays. The two liquid crystal materials which are commonly used in display technology are nematic and cholesteric whose schematic arrangement of molecules is shown in fig. The most popular liquid crystal structure is the Nematic Liquid Crystal (NLC).

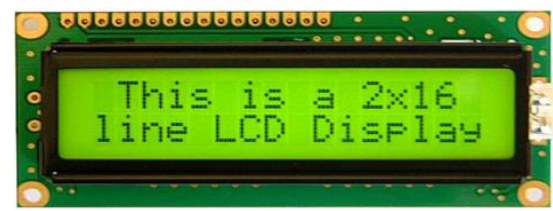

Fig.8. LCD Display.

In this all the molecules align themselves approximately parallel to a unique axis (director), while retaining the complete translational freedom. The liquid is normally transparent, but if subjected to a strong electric field, disruption 
of the well ordered crystal structure takes place causing the liquid to polarize and turn opaque. The removal of the applied electric field allows the crystal structure to regain its original form and the materials become transparent.

\section{CONCLUSION}

This project proposed a localization system for a mobile robot with the aid of RFID technology and an optimized algorithm. A sensor model was built based on RFID technology by using low frequency to estimate the distance between RFID tags and the mobile robot. The effect of different orientations between the RFID reader and the RFID tag on LF was analyzed, and the result shows that orientation does not have a significant effect in this project. A robotic system for localizing paths of an unknown warehouse has been proposed. The system resorts to low cost technology as a unicycle-like vehicle with a differential drive kinematics and off-the-path RFID reader and tags. It estimates the position of the tag using a two steps procedure: first, a Kalman-based algorithm permits the robot to localize itself. Then, the robot estimates the position of tagged objects by matching the phase pattern collected along specific paths to that of an electromagnetic model.

\section{FUTURE WORK}

On the other hand, multipath is a deterministic phenomenon that changes with the relative position among the reader, tags and scattering paths so that, collecting a number of phase measurements sufficiently large, it is possible to include data with little multipath. The proposed matching algorithm takes advantage from the multipath-free data at some measurements points to attenuate the disguising effects of the multipath at other points. Future research could be devoted to the development of a localization algorithm able to select multipath-free data (or low multipath-affected data) from the set of measurements in order to improve the accuracy of localization. Also the most common applications are related to the exploration of unknown and/or dangerous spaces that are not accessible to people by exploiting the advantages offered by ultrasonic technology. Also our future work includes deployment and test of the sensor network in different environments and different industrial application configurations.

\section{REFERENCES}

[1]. D. B. Jourdan, D. Dardari, and M. Z. Win, "Position error bound for UWB localization in dense cluttered environments," IEEE Trans. Aerosp. Electron. Syst., vol. 42, no. 2, pp. 613-628, Apr. 2008.

[2]. H. Liu, H. Darabi, P. Banerjee, and J. Liu, "Survey of wireless indoor positioning techniques and systems," IEEE Trans. Syst., Man, Cybern. C, Appl. Rev., vol. 37, no. 6, pp. 1067-1080, Nov. 2007.

[3]. C.-H. Chang, S.-C. Wang, and C.-C. Wang, "Exploiting moving objects: Multi-robot simultaneous localization and tracking," IEEE Trans. Autom. Sci. Eng., vol. 13, no. 2, pp. 810-827, Apr. 2016.

[4]. K. Finkenzeller, RFID Handbook: Radio-Frequency Identification Fundamentals and Applications. Hoboken, NJ, USA: Wiley, 2000.

[5]. S. Park and S. Hashimoto, "Autonomous mobile robot navigation using passive RFID in indoor environment," IEEE Trans. Ind. Electron., vol. 56, no. 7, pp. 2366-2373, Jul. 2009

[6]. A. Nubiola and I. A. Bonev, "Absolute calibration of an abb irb 1600 robot using a laser tracker," Robotics and Computer-Integrated Manufacturing, vol. 29, no. 1, pp. 236-245, 2013.

[7]. Y. M. Zhao, Y. Lin, F. Xi, and S. Guo, "Calibration-based iterative learning control for path tracking of industrial robots," IEEE Transactions on Industrial Electronics, vol. 62, no. 5, pp. 2921-2929, 2015.

[8]. W. J. Wilson, C. C. W. Hulls, and G. S. Bell, "Relative end-effector control using cartesian position based visual servoing," IEEE Transactions on Robotics and Automation, vol. 12, no. 5, pp. 684-696, 1996.

[9]. F. Janabi-Sharifi, L. Deng, and W. J. Wilson, "Comparison of basic visual servoing methods," IEEE/ASME Transactions on Mechatronics, vol. 16, no. 5, pp. 967-983, 2011.

[10]. M. Keshmiri and W.-F. Xie, "Image-based visual servoing using an optimized trajectory planning technique," IEEE/ASME Transactions on Mechatronics, vol. 22, no. 1, pp. 359-370, 2017.

[11]. E. L. Berz, D. A. Tesch and F. P. Hessel, "RFID indoor localization based on support vector regression and k-means," Proceedings of the 24th IEEE International Symposium on Industrial Electronics (ISIE 2015), pp. 1418-23, Buzios (Rio de Janeiro, Brazil), June 3-5, 2015.

[12]. J. S. Choi, H. Lee, D. W. Engels, and R. Elmasri, "Passive UHF RFIDbased localization using detection of tag interference on smart shelf," IEEE Transactions on System, Man, Cybernetics, Part C (Applications \& Reviews), vol. 42, no. 2, pp. 268-275, Mar. 2012.

[13]. J. Wang, F. Adib, R. Knepper, D. Katabi, and D. Rus, "RF-compass: Robot object manipulation using RFIDs," Proceedings of MOBICOM 2013, Miami (Florida), Sept. 30 - Oct. 4, 2013.

[14]. H. Y. Yu, J. J. Chen and T. R. Hsiang, "Design and Implementation of a Real-Time Object Location System Based on Passive RFID Tags," IEEE Sensors Journal, vol. 15, no. 9, pp. 5015-5023, Sept. 2015. 